\title{
Research on Surveying and Mapping Technologies of UAV Laser
}

\author{
Zhizhang Wan ${ }^{1}$, Ping Gan ${ }^{1}$ \\ ${ }^{1}$ Nanchang Institute of Science \& Technology, Nanchang, 330108, China
}

Keywords: UAV laser; surveying and mapping technology; unmanned aerial vehicle

\begin{abstract}
The laser scanner can quickly scan the measurement area, greatly saving the time and cost of the operation. The small UAV is highly efficient in aerial photography and convenient for data processing in the industry. In the harsh terrain area, a supplement and accuracy check for the missed area of the scanner can be conducted. This paper describes the key technologies of UAV laser of information acquisition, information processing and wind-resistance protection and gives the applications of surveying and mapping technologies of UAV laser in the industries of geological exploration, mobile communication and electric power to provide some references for the relative researchers.
\end{abstract}

\section{Introduction}

The UAV with its advantages in mountainous terrain mapping applications can quickly obtain the measured image [1]. The UAV does not exist and measuring blind area, point cloud processing is simple and fast, a lot of open source software, including scanner software for UAV postprocessing can point cloud processing. In commercial applications, there have also been many UAV agricultural medicine, UAV traffic inspection, unmanned aerial vehicle express and other applications. To sum up, the current UAV technology has entered a mature stage as a flying carrier. It can provide a stable flight platform for all walks of life, so that it can be applied in many ways. Our country city to speed up construction progress in economic construction, the construction of the number of construction projects, construction scale is expanding constantly, in the project construction process, need to use advanced technology and equipment to engineering measurement, to provide the necessary conditions for the construction of the construction project. In the new era, the research and application of science and technology is deepening. The technology of Surveying and mapping applied in engineering survey is constantly evolving and intelligent zed, which greatly improves the accuracy of engineering data measurement. A technology which is widely applied in engineering surveying and mapping is UAV mapping technology. UAV mapping technology has prominent effect, and can accomplish various measurement tasks in different environments. The ground 3D laser scanner can solve the problem of mountain steep dangerous terrain scanning with its flexible, fast and non-contact measurement method. And it can get high-precision measurement results. In actual engineering applications, the situation of survey area is often very bad. There are many vegetation and deep valleys in mountainous area. The scanning progress is slow, and the effect of three-dimensional laser on vegetation filtering is limited. Unmanned aerial vehicle aerial survey is widely used in small scale and large-scale mapping with its advantages of easy operation and flexible maneuver [2].

\section{Key Technologies of Surveying and Mapping of UAV Laser}

\subsection{Information Acquisition Technology}

After a general understanding of the survey area, the original point cloud data of the measured area was obtained through the sweeping survey, and the site coordinates were measured by the measurement. After the station registration and stitching of measured point cloud data is complete, the vegetation filtering of point cloud, obtain geomorphic data, the establishment of DEM and the 
drawing of contour line: in the survey area under harsh conditions, the scanning process, the terrain constraints, point cloud scanning the existing problem of high precision processing the data is not complete, dense vegetation: in view of the above problems, the UAV aerial way up. The use of UAV mapping technology requires a variety of information on construction projects were collected in engineering surveying, data collected by different subjects, there is no work involved in the application of man-machine mapping technology is different, can be divided into automatic acquisition encryption and manual acquisition part, manual data collection requires the use of computer technology and equipment and other facilities remote control, UAV mapping and technical personnel in the base station according to the actual needs of engineering data, the operation of UAV image shooting, image content and selectively targeted, make the data more practical. Using UAV mapping technology to automatically encrypt is a way to protect and control the internal system, which can make information storage safe and ensure data collection and application stable. R \& D personnel to actively carry out R \& D work and do the market research, research and development based on customer needs, establish and perfect the system of customer service mechanism, actively establish technology research and development, operation and maintenance, technical training, customer service and technical updates and other one-stop services, establish and improve the UAV laser mapping measurement system research and the application of network [3].

\subsection{Information Processing Technology}

The first step of the motion recovery structure algorithm is to extract the feature points of the UAV and to match the high precision image. First, feature extraction operators are used to extract the feature points of each unmanned aerial vehicle. Secondly, the approximate nearest neighbor based on the $\mathrm{K}$ - D tree is used. Then, we use the algorithm based on the random extraction consistency framework to compute the basic matrix to eliminate the mismatching points, and establish the set of feature points that satisfy the polar geometric constraints to complete the matching of the same name feature points. The real-time monitoring of the project information and the surrounding circumstances using UAV mapping technology, to obtain the relevant data after the need for data processing, data processing in the past is pure artificial form, so data quality and processing speed is difficult to guarantee. Computer mapping technology application, the comprehensive analysis of the overall project and geographical environment, to the ecological situation of aerial project surrounding the image content with aerial shooting and low altitude shooting, data analysis, discover the problem lies in the macro guidance, then in data transmission, to develop effective strategies to solve the UAV project measurement data processing speed and efficiency. This system can obtain high resolution and high-resolution image data under low altitude conditions, and meet the needs of large scale mapping. This system is equipped with ultra-wide self-calibration with low altitude digital camera system, through the unique design of the calibration software and error correcting image overlap caused by mechanical deformation. The accuracy error caused by the deflection of the camera attitude angle is made up by the edge field. At the same time, the system greatly reduces the weight of the imaging system, and meets the needs of unmanned and low altitude aerial survey [4].

\subsection{Wind-resistance Protection Technology}

The unmanned aerial vehicle (UAV) laser mapping can obtain automatic flight planning and flight photography, reducing a large amount of manual work. At the same time, the flying platform of UAV is very easy to take off and land, so that the UAV surveying and mapping can adapt to the surveying and mapping tasks and needs of various terrain and regions. UAV surveying and mapping technology in the engineering survey, need to be taken into account in the jungle, mountain area and the special environment use effect, engineering measurement, relevant personnel for UAV landing should be set reasonably, so that it can successfully fly in reasonable glide distance; for large UAVs, because the reality of the situation may not be able to meet the flight conditions, taking into account the natural environment attribute, adjust take-off height, improve the wind resistance, prevent the UAV crashed due to mountain wind disturbance. In addition, in the engineering measurement of UAV application mapping technology, need to consider whether the mechanical equipment shape and weight affect the 
off state, combined with the actual situation, the parameters of the UAV control equipment, improve the technical safety, meet the actual demand of Engineering surveying. Data editing module is mainly responsible for the massive data editing, data management algorithm is efficient in memory and external memory. This reference to the real-time visualization of large scale terrain algorithm based on the tree, the algorithm first data space into blocks, further space partition block. The data filter module data acquisition module is mainly responsible for the postprocessing of point cloud data reference cloud data filtering method of virtual network and three corner points based on the gradient filter. The method of virtual triangulation and slope filtering combining point cloud data processing, the concept of virtual triangulation for point cloud filtering, avoid the point cloud smoothing caused the loss of information.

\section{Applications of Surveying and Mapping Technologies of UAV Laser}

\subsection{Geological Exploration Applications of Surveying and Mapping Technologies of UAV Laser}

The UAV laser mapping system to construct topographic technology for surveying management has brought great convenience degree. The UAV laser mapping system topographic mapping technology in the process of continuous application, can effectively play the role of snapshot and real, can the actual situation of engineering geological exploration, records accurately, and improve the resolution of the image to the greatest extent. Therefore, the UAV laser mapping system topographic map in the geological survey work, play an important role and significance. Among them, there are the main contents: the management environment changes, land salinization and other content, so the relevant leaders can according to images and information, for the full deployment of staff to ensure the balance of the engineering geological environment to the maximum extent, can effectively ensure the geological prospecting work in an orderly manner. Because of the relatively lack of water resources in China, the exploration of underground water by geological engineering is particularly important. Therefore, in the process of UAV laser mapping system topographic mapping technology application in underground water is a very important work, but in the traditional survey techniques, and not on the subsurface waters of the situation and information. However, in the process of continuous development of UAV laser mapping system topographic mapping technology and application of underground water and can balance the degree of overall acquisition, the staff in the use of computer technology in the form of the relevant information and image analyze and organize, which can meet the social needs of our country the water in the maximum extent. In addition, in the process of UAV laser mapping system topographic mapping technology application in the form of underground water can change timely grasp and update, and the related information and images.

\subsection{Mobile Communication Applications of Surveying and Mapping Technologies of UAV Laser}

No laser inspection system is a man-machine communication, aviation, image recognition, remote sensing, global positioning system, geographic information system and many other advanced technologies in one of the complex inspection system, due to the low altitude UAV has the advantages of small volume, low cost, real-time and flexible and efficient, can easily climb mountains, across the river. The unmanned aerial vehicle can observe the low altitude objects from many angles. Using UAV to carry out daily safety inspection of communication base stations and antennas, instead of traditional manpower inspection, can reduce human potential risks in inspection process and reduce property losses of enterprises. Secondly, the field environment is complex, many regional survey personnel cannot reach, UAV remote sensing combined with vehicle control technology, remote sensing technology, GPS technology, geographic information system technology. The equipment control of UAV image sensor reconnaissance aircraft arrived in the area to carry control, camera equipment automatic control camera to obtain high resolution image tilt photography, GPS technology, real-time monitoring of UAV laser location, UAV position information 
visualization technology of dynamic geographic information system. The ground staff only need to control the position of UAV. The tilting photogrammetry post-processing software can process the image data and get the accurate and detailed basic geographic information data around the exploration area, and provide reliable geographic information data for the base station construction. In addition, the optimization on the ground network test only the traditional UAV equipped with laser can improve test equipment height of communication signals in different locations around the base station network test, broke through the highly restricted optimization personnel can obtain the test data with the network, the optimization of communication signal base station network, the network signal coverage area is smoother.

\subsection{Electric Power Applications of Surveying and Mapping Technologies of UAV Laser}

The power line design is to design an optimal power transmission line according to the relevant design principles. According to the requirements, a qualified power transmission line is designed, and we need to know the geographical environment information in detail. To ensure the safe operation of power transmission lines, it is very important for the electric power department to inspect the power transmission lines regularly. Power line inspection refers to the inspection of transmission channel, tower rod and transmission component, and the hidden danger of transmission equipment is found in time. The traditional inspection work mainly relies on manual work, the field work environment is bad and the labor intensity is big and the efficiency is low. In dangerous areas such as cliffs and swamps, the life and safety of patrol personnel are threatened. When there are natural disasters such as earthquakes, landslides and floods, patrol personnel will not be able to carry out inspections. Compared with other circuit inspection methods, UAV flight airspace and air traffic control are low, no restrictions, not affected by climate, geographical environment, simple operation, easy to grasp, uninterrupted work characteristics, therefore, low altitude UAV inspection can well solve these problems. The UAV line inspection has two main application modes, one is the camera mounted on the UAV pod, only one person to operate the UAV, post videos view transmission line; two is real-time and UAV data communication, real-time image transmission to the ground monitor screen shot, the control of one person UAV, a person watching video, two people completed with each other on line inspection, if found suspicious characteristics control observation without careful examination of human hover, power transmission equipment, and security risks exist. In addition, the UAVs can also carry different imaging devices to observe the transmission components, such as the infrared thermal imager can detect the temperature rise of the components. The UAV patrol line to reduce work intensity of laser line patrol workers, reduce the transmission line maintenance cost, no performance superior to become efficient man-machine tool power line inspection, UAV laser transmission line inspection will be an efficient and promising way of inspection.

\section{Conclusion}

UAV mapping technology has been applied in many fields. The state has increased the research of UAV mapping technology, enabling it to play the biggest advantage in the civilian field. The UAV laser can quickly obtain different geographic information and improve the speed of data processing. When we are using UAV laser technology, we should conduct directional analysis in data acquisition, and get high-definition mapping images in time to ensure the safety of operations.

\section{References}

[1] Li Mousi, Sun Yu, Wei Rongyi, He Baogen. Application of Combining the 3D Laser Measurement System and the Unmanned Aerial Vehicle in Mountainous Area Surveying [J]. Pearl River, 2017, 38(5): 23-26.

[2] Luo Dongshan, He Jun, Cui Lishui. Design and Realization of UAV Airborne Laser Scanning Measurement System [J]. Geomatics \& Spatial Information Technology, 2015, 38(10): 175-177. 
[3] Li Quanzhou, Shi Gaofeng, Cui Jianjun. Overview of UAV surveying and mapping remote sensing [J]. Telecom Engineering Technics and Standardization, 2017, 30(4): 14-17.

[4] Lv Limei. Application Research of UAV Aerial Photography Technology in Large Scale Mapping [J]. Geomatics \& Spatial Information Technology, 2016, 39(2): 116-118+122. 